\title{
Nanoscale
}

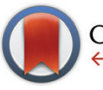

CrossMark \&lick for updates

Cite this: Nanoscale, 2015, 7, 14822

Received 26th June 2015,

Accepted 13th August 2015

DOI: $10.1039 / c 5 n r 04273 a$

www.rsc.org/nanoscale

\section{Formation of long single quantum dots in high quality InSb nanowires grown by molecular beam epitaxy}

\author{
Dingxun Fan, ${ }^{a}$ Sen Li, ${ }^{a}$ N. Kang, ${ }^{\star a}$ Philippe Caroff,,$\dagger^{b}$ L. B. Wang, ${ }^{a}$ Y. Q. Huang, ${ }^{a}$ \\ M. T. Deng, ${ }^{c}$ C. L. $Y u^{c}$ and H. Q. Xu*a,c
}

\begin{abstract}
We report on realization and transport spectroscopy study of single quantum dots (QDs) made from InSb nanowires grown by molecular beam epitaxy (MBE). The nanowires employed are 50-80 $\mathrm{nm}$ in diameter and the QDs are defined in the nanowires between the source and drain contacts on a $\mathrm{Si} / \mathrm{SiO}_{2}$ substrate. We show that highly tunable $Q D$ devices can be realized with the MBE-grown InSb nanowires and the gate-to-dot capacitance extracted in the many-electron regimes is scaled linearly with the longitudinal dot size, demonstrating that the devices are of single InSb nanowire QDs even with a longitudinal size of $\sim 700 \mathrm{~nm}$. In the few-electron regime, the quantum levels in the QDs are resolved and the Landé $g$-factors extracted for the quantum levels from the magnetotransport measurements are found to be strongly level-dependent and fluctuated in a range of 18-48. A spin-orbit coupling strength is extracted from the magnetic field evolutions of a ground state and its neighboring excited state in an InSb nanowire QD and is on the order of $\sim 300 \mu \mathrm{eV}$. Our results establish that the MBE-grown InSb nanowires are of high crystal quality and are promising for the use in constructing novel quantum devices, such as entangled spin qubits, one-dimensional Wigner crystals and topological quantum computing devices.
\end{abstract}

Over the past decade, transport measurements of semiconductor quantum dots (QDs) have been widely used for exploring novel physics and new applications. Many-body phenomena, such as the Kondo effect ${ }^{1-5}$ and solid-state spin qubits, ${ }^{6,7}$ have been extensively studied using semiconductor QDs. These abundant phenomena rely on the coherent nature of electron transport in the systems. More recently, InSb nanowires (NWs) have attracted an increasing interest..$^{8-18}$ Owing to the intrinsic

\footnotetext{
${ }^{a}$ Department of Electronics and Key Laboratory for the Physics and Chemistry of Nanodevices, Peking University, Beijing 100871, China. E-mail: nkang@pku.edu.cn, hqxu@pku.edu.cn

${ }^{b}$ I.E.M.N., UMR CNRS 8520, Avenue Poincaré, BP 60069, F-59652 Villeneuve d'Ascq, France

${ }^{c}$ Division of Solid State Physics, Lund University, Box 118, S-221 00 Lund, Sweden $\dagger$ Present address: Department of Electronic Materials Engineering, Research School of Physics and Engineering, the Australian National University, Canberra, ACT 0200, Australia.
}

properties of bulk InSb, such as a small bandgap $E_{\mathrm{g}}=0.17 \mathrm{eV}$, a high electron mobility $\mu_{\mathrm{e}}=77000 \mathrm{~cm}^{2} \mathrm{~V}^{-1} \mathrm{~s}^{-1}$, a small electron effective mass $m_{\mathrm{e}}^{*}=0.015 m_{\mathrm{e}}$ (where $m_{\mathrm{e}}$ is the bare electron mass), and a large electron Landé $g$ factor $\left|g^{*}\right|=51,{ }^{19,20} \mathrm{InSb}$ nanowires have potential applications in the fields of quantum computation, spintronics and high-speed electronics. Using state-of-the-art nanofabrication techniques, various InSb NW devices, such as field-effect transistors, ${ }^{10,13,17}$ single and double QDs, ${ }^{8-10,12,16}$ and semiconductor-superconductor hybrid quantum devices, ${ }^{11}$ have been realized. Studies of these devices have led to the observations of phase-coherent universal conductance fluctuations, ${ }^{13}$ large and energy level-dependent $g$-factors, ${ }^{8,9,14}$ strong spin-orbit interaction strengths, ${ }^{8,12}$ and correlation-induced conductance suppression, ${ }^{9}$ and to the demonstration of electric manipulation of electron and hole spin states. ${ }^{12,15}$ Very recently, Majorana bound states in solid state systems have attracted great attention, because of their potential applications in topological quantum computing, and InSb semiconductor NW-superconductor hybrid quantum devices have been developed to spot the signatures of these exotic, topologically distinctive states. ${ }^{21-24}$ Nevertheless, in the aforementioned works, the InSb NWs used have primarily been grown by metal-organic vapor phase epitaxy (MOVPE) or chemical vapor deposition (CVD). Due to their intrinsically high material purity and crystal quality, InSb nanowires grown by molecular beam epitaxy (MBE) are desired for the development of well-defined quantum and topological devices to detect novel, exotic physics phenomena. However, electrical properties of MBE-grown InSb NWs have only been studied at room temperature. $^{25}$ Quantum devices made from these presumably high-quality MBE-grown InSb NWs and many-body coherent transport in such devices have yet to be demonstrated.

In this communication, we report on the realization and low-temperature transport measurements of single QDs in MBE-grown InSb NWs. The measurements of the QD devices in the many-electron regime demonstrate regular, consecutive Coulomb diamonds of similar sizes in the charge stability diagrams, indicating that the transport occurs dominantly as single electron tunneling through the QDs. The gate capaci- 
tance to the QDs deduced from Coulomb oscillations is found to scale linearly with the spacing between the source and drain contacts, demonstrating that the devices are of single QDs and each is built from the entire nanowire segment between the contacts. In the few-electron regime, the devices are studied by magnetotransport spectroscopy measurements. The $g$ factors are extracted for different quantum levels in the QDs using magnetic-field evolutions of both the differential conductance at different back-gate voltages and the linear-response conductance at zero bias voltage. The measured $g$ factors show leveldependent fluctuations in a range of 18-48, indicating the presence of spin-orbit interaction in the InSb NW QDs. A spin-orbit coupling strength of $\sim 275 \mu \mathrm{eV}$ is extracted from the magnetic-field evolutions of a ground state and its neighboring excited state of an InSb NW QD in this work.

All InSb nanowires used in this study are grown by gassource MBE using gold seed particles decorating an InP(111)B substrate (Fig. 1a). The gold seeds are obtained by dewetting of a $0.3 \mathrm{~nm}$ nominally thick gold film at $510{ }^{\circ} \mathrm{C}$. Growth is initiated by growing homoepitaxial InP wurtzite stem nanowire segments for $15 \mathrm{~min}$, followed by growing wurtzite InAs stem segments for $25 \mathrm{~min}$, before switching to grow InSb nanowires for $25 \mathrm{~min}$. Cooling down is performed with a linearly decreasing $\mathrm{Sb}_{2}$ molecular flux during $45 \mathrm{~s}$ and then in vacuum until the sample is removed from the growth chamber at room temperature. The $\mathrm{Sb}_{2}$ flux is obtained from a Veeco valvedcracker cell. The growth is performed at $410{ }^{\circ} \mathrm{C}$ for all segments. The In flux $2 \mathrm{D}$ equivalent growth rate is calibrated to $0.50 \mathrm{ML} \mathrm{s}^{-1}$ on an $\operatorname{InAs}(001)$ buffer layer and the total V/III ratio (estimated by the ratio of $2 \mathrm{D}$ equivalent growth rates for both group III and group V adatoms) is set to 1.95 for the InSb segment growth. A perfect twin-free zincblende crystal structure is obtained independently of diameter for all InSb nanowire segments. Crystal phase perfection over a large range of growth conditions, nanowire dimensions, or growth techniques, is a unique advantage of the gold-seeded antimonide nanowire family, which has been confirmed independently in several works. $^{26}$ Our InSb nanowire segments are also completely free of tapering. See previous works focused on the growth and structural properties for more details on these aspects. ${ }^{9,25,27}$

The as-grown InSb NWs are mechanically transferred onto an $\mathrm{n}^{++}$doped Si substrate covered by $105 \mathrm{~nm}$ thick thermally grown $\mathrm{SiO}_{2}$, which serve as a global back gate and gate dielectric, respectively. Standard electron-beam lithography is used to define source and drain electrodes and to connect the electrodes and thus nanowires to pre-defined outer bonding pads. After development, the sample is oxygen-plasma ashed for $15 \mathrm{~s}$ to remove the resist residues and then chemically etched in a diluted $\left(\mathrm{NH}_{4}\right)_{2} \mathrm{~S}_{x}$ solution at $35{ }^{\circ} \mathrm{C}$ for $1 \mathrm{~min}$ to remove the native surface oxide. ${ }^{28}$ Then the sample is immediately loaded into the vacuum chamber, followed by thermal evaporation of $\mathrm{Ti} / \mathrm{Au}(5 / 100 \mathrm{~nm})$ as contact metal and lift-off in acetone and isopropanol (IPA). A quantum dot is formed in an InSb NW segment between the contacts by naturally formed Schottky barriers at cryogenic temperatures.

Fig. 1b shows a tilted, false-colored SEM image of a fabricated InSb NW QD device. The diameters of NWs used in our device fabrication are in a range of 50-80 $\mathrm{nm}$. The fabricated devices are characterized at room temperature in a probe station. Devices with two-terminal resistances of 20-100 k $\Omega$ are selected and cooled down in a ${ }^{3} \mathrm{He} /{ }^{4} \mathrm{He}$ dilution refrigerator for low-temperature transport spectroscopy studies. The data are recorded using both dc and ac measurement methods in different cooling-down cycles. In the dc measurements, the voltage is applied antisymmetrically to the source and drain electrodes to suppress common-mode noise. The current is amplified using a home-made current amplifier and is numerically differentiated to obtained the conductance. While in the ac measurements, a $17.3 \mathrm{~Hz}, 5 \mu \mathrm{V}$ root-mean-squared excitation voltage source is fed into one of the two contacts of a device and the current is recorded from the other side. The signal lines are carefully filtered and all the measurements are carried out at a base temperature of $60 \mathrm{mK}$. In the magnetotransport measurements, the magnetic field $B$ is applied perpendicularly to the substrate and thus to the NWs in the devices.
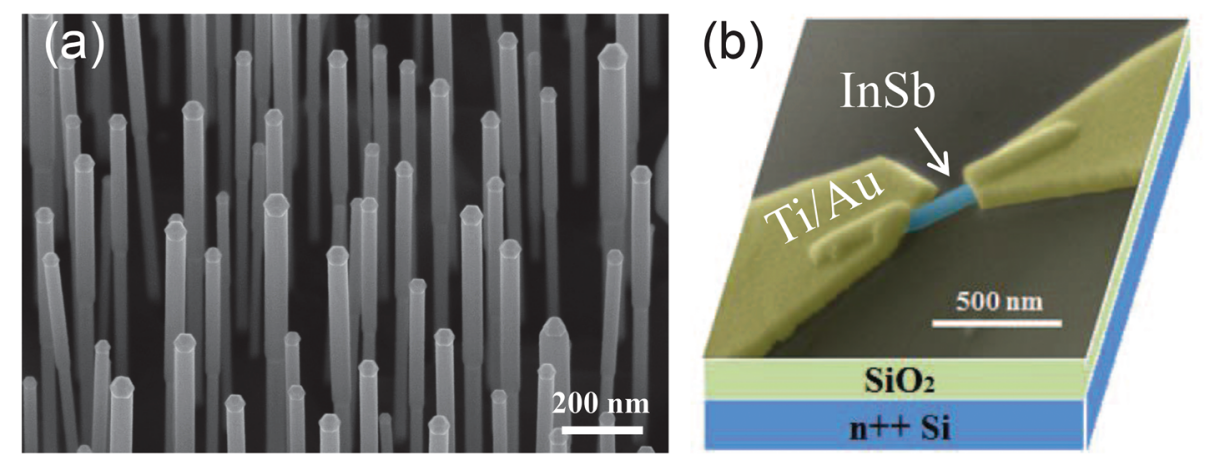

Fig. 1 (a) Representative SEM image ( $30^{\circ}$ tilted angle view) of the as-grown nanowire sample, illustrating from top to bottom the Auln ${ }_{2}$ seed particles, the top InSb segments, and the thinner InAs stems, respectively. (b) SEM image (in false colors) of a typical InSb nanowire device contacted by $\mathrm{Ti} / \mathrm{Au}$ and schematic view of the $\mathrm{Si} / \mathrm{SiO}_{2}$ substrate on which the device is fabricated. An InSb nanowire quantum dot is formed between the two $\mathrm{Ti} / \mathrm{Au}$ contacts at low temperatures. 
Fig. 2a shows the two-terminal current $I_{\mathrm{ds}}$ of a device with $\sim 68 \mathrm{~nm}$ in the NW diameter and $\sim 391 \mathrm{~nm}$ in the contact spacing measured as a function of the back gate voltage $V_{\mathrm{bg}}$ at a fixed bias voltage of $V_{\mathrm{ds}}=100 \mu \mathrm{V}$. It is seen that the measured current $I_{\mathrm{ds}}$ shows regular, nearly periodic current oscillations with a series of sharp peaks separated by zero conductance regions, i.e., the single-electron Coulomb blockade (CB) effect in the many-electron regime. We estimate the gate capacitance $C_{\mathrm{g}}$ from the averaged peak-to-peak separation, which is solely determined by the capacitive coupling of the QD to the gate, and find $C_{\mathrm{g}} \approx 16 \mathrm{aF}$. Fig. $2 \mathrm{~b}$ shows the differential conductance $\mathrm{d} I_{\mathrm{ds}} / \mathrm{d} V_{\mathrm{ds}}$ of the device measured as a function of $V_{\mathrm{ds}}$ and $V_{\mathrm{bg}}$ (charge stability diagram). Here, the differential conductance is represented in a color scale with bright (dark) color corresponding to high (low) conductance. Fig. 2b consists of 14 consecutive, diamond-shaped, dark areas in which the charge transport is prohibited due to the single-electron CB effect. The sizes of the diamonds do not show clear electron-number dependence, indicating negligible contribution of the quantization energy $\Delta \varepsilon$ to the single-electron addition energy $\Delta E$ to the QD, where in the constant interaction model $\Delta E=\Delta \varepsilon+E_{\mathrm{C}}$ and $E_{\mathrm{C}}$ is the single-electron electrostatic charging energy. Hence, we extract from the vertical sizes of the diamonds an averaged charging energy $E_{\mathrm{C}} \sim \Delta E \sim 3.5 \mathrm{meV}$, which gives an averaged value for the total QD capacitance $C_{\Sigma}$ of $45 \mathrm{aF}$ and an averaged value for the gate lever arm factor $\alpha=C_{\mathrm{g}} / C_{\Sigma}$ of 0.36 . Indeed, using an ideal one-dimensional infinitely deep quantum well model, the longitudinal single-particle quantiza-
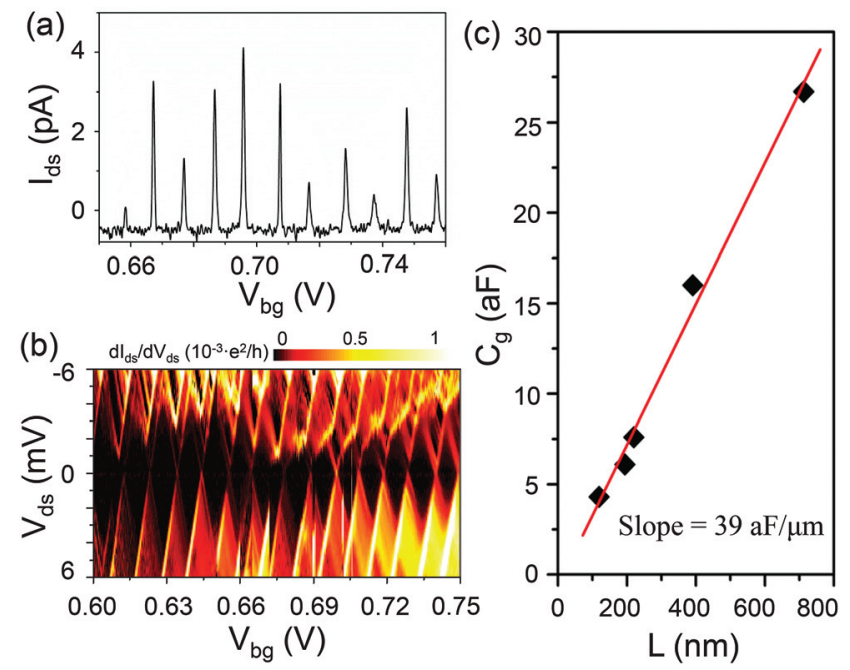

Fig. 2 (a) Coulomb blockade oscillations of the source-drain current measured for an InSb nanowire quantum dot device with a spacing of $391 \mathrm{~nm}$ between the source and drain contacts at applied bias voltage $V_{\mathrm{ds}}=0.1 \mathrm{mV}$. (b) Plot of the differential conductance as a function of back gate voltage $V_{\mathrm{bg}}$ and bias voltage $V_{\mathrm{ds}}$ (charge stability diagram) for the InSb nanowire quantum dot in the many-electron regime. (c) Gate capacitance extracted as a function of the source-drain spacing $L$. The red line with a slope of $39 \alpha \mathrm{F} \mu \mathrm{m}^{-1}$ is a linear fit to the data points extracted from the measurements of five InSb nanowire quantum dot devices. tion energy in the nanowire with a length of $L=391 \mathrm{~nm}$ is $\Delta \varepsilon$ $\sim 0.4 \mathrm{meV}$, an order of magnitude smaller than the charging energy. In addition, one can also observe that the sizes of the CB diamonds are slightly smaller in the higher $V_{\mathrm{bg}}$ region, possibly due to an increase in the QD size at more positive $V_{\mathrm{bg}}$.

The regular, consecutive $\mathrm{CB}$ diamond structures seen in Fig. 2b suggest the formation of a single quantum dot in the device. Existence of more dots in series in the device would be expected to show a more complicated overlapping diamond structure in the charge stability diagram. ${ }^{29-31}$ This result of the formation of a single quantum dot in a $391 \mathrm{~nm}$-long MBEgrown InSb NW is very encouraging. To support the result, the gate-to-dot capacitances $C_{\mathrm{g}}$ of four other fabricated InSb NW devices are measured. These devices are made from the InSb nanowires of similar thickness (70-80 $\mathrm{nm}$ in diameter) but have different source-to-drain contact spacings and the measurements are all performed in the well-defined manyelectron regime in which 10 or more consecutive current peaks with an approximately equal spacing are observed in each device. Fig. 2c shows the gate-to-dot capacitance $C_{\mathrm{g}}$ extracted from the measurements as a function of the source-to-drain spacing $L$. It is seen that the measured data points are fitted reasonably well to a straight line with a slope of $39 \pm 2$ aF $\mu \mathrm{m}^{-1}$, indicating the formation of a single QD in each of these devices, including the one with the source-to-drain spacing of $\sim 700 \mathrm{~nm}$. Note that we have also estimated the gate capacitance $C_{\mathrm{g}}$ to an InSb nanowire segment using a cylinder-onplane model ${ }^{32}$ and found values of $C_{\mathrm{g}}$ which are only $20 \%-$ $30 \%$ higher than the corresponding experimental data. Here, the deviations could be understood as the combined effect of screening from the source and drain electrodes and of neglecting the contribution from small but finite quantum level spacings. We would like to emphasize again that these data demonstrate that the QD size is solely determined by the spacing between the source and drain contacts, underscoring the high crystal quality of the MBE-grown InSb nanowires. We also note that the effects of lattice defects, crystallographic imperfections, or impurities in the nanowires, which could break a single QD into a multiple one, have not been observed in these measurements.

Having demonstrated the electron transport though single QDs in the MBE-grown InSb NW devices in the many-electron regime, we now move on to the investigation of the transport properties of the devices in the few-electron regime. Fig. 3a presents the measured stability diagram of a QD device made with a NW of $\sim 74 \mathrm{~nm}$ in diameter and a source-drain spacing of $L \sim 120 \mathrm{~nm}$. Signals along $V_{\mathrm{ds}}=0$ drop to the noise floor for $V_{\mathrm{bg}}<1.28 \mathrm{~V}$, although it is not the case of the last electron occupation on the dot. The addition energy exhibits large variations, from $\sim 3 \mathrm{meV}$ to $\sim 10 \mathrm{meV}$, which can be attributed to large level quantization in the few-electron regime. In the gate voltage region of $1.34 \mathrm{~V}<V_{\mathrm{bg}}<1.49 \mathrm{~V}$, the transport is characterized by a distorted Coulomb diamond. In particular, a sawtooth structure is seen to appear at the edge of the diamond. This saw-tooth structure, which is more clearly seen on the negative bias voltage side, could most likely be the effect of the 

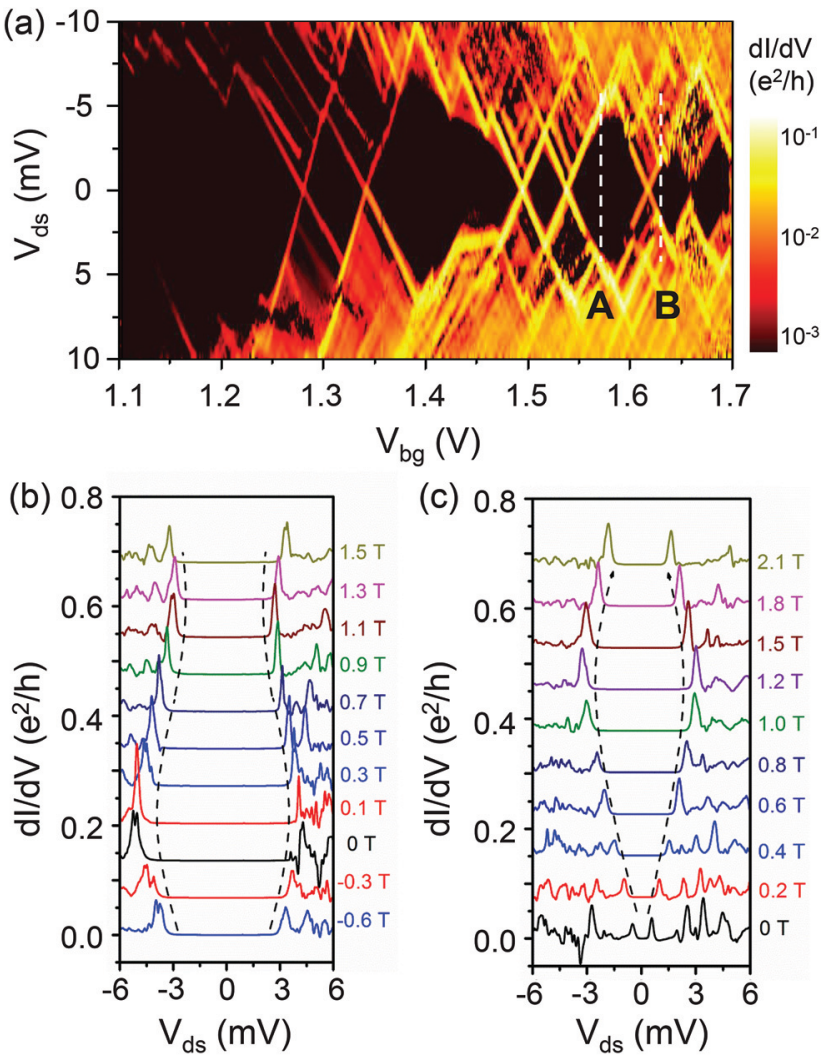

Fig. 3 (a) Charge stability diagram of an InSb nanowire quantum dot device with a source-drain spacing of $\sim 120 \mathrm{~nm}$ in the few-electron regime. (b) Magnetic-field evolution of the differential conductance $\mathrm{d} l_{\mathrm{ds}} / \mathrm{d} V_{\mathrm{ds}}$ measured for the device at back gate voltage $V_{\mathrm{bg}}=1.57 \mathrm{~V}$, i.e., along the white-dashed line cut $A$ in (a). (c) Magnetic-field evolution of the differential conductance $\mathrm{d} l_{\mathrm{ds}} / \mathrm{d} V_{\mathrm{ds}}$ measured for the device at back gate voltage $V_{\mathrm{bg}}=1.62 \mathrm{~V}$, i.e., along the white-dashed line cut $\mathrm{B}$ in (a). The dashed lines in (b) and (c) are guides to the eyes.

population of remote, localized charge traps surrounding the $\mathrm{QD}$, e.g., in the gate dielectrics. Horizontal features seen clearly inside this distorted Coulomb diamond are signs of cotunneling involving an excited state.

In order to extract the spin properties of quantum levels in the MBE-grown NW QD, we show, in Fig. 3b and c, the evolution of the differential conductance $\mathrm{d} I_{\mathrm{ds}} / \mathrm{d} V_{\mathrm{ds}}$ of the device with increasing magnetic field at two fixed gate voltages $V_{\mathrm{bg}}=$ $1.57 \mathrm{~V}$ and $1.62 \mathrm{~V}$, i.e., along the white dashed line cuts $\mathrm{A}$ and $\mathrm{B}$ in Fig. 3a. At $V_{\mathrm{bg}}=1.57 \mathrm{~V}$, the QD is occupied by an even number of electrons in the $\mathrm{CB}$ region and the pair of inner $\mathrm{d} I_{\mathrm{ds}} / \mathrm{d} V_{\mathrm{ds}}$ peaks, sitting symmetrically around $V_{\mathrm{ds}}=0$ as seen in Fig. $3 \mathrm{~b}$, flags the transport through the two last occupied spindegenerate orbital levels. With increasing $B$, the two peaks start to move towards each other, reflecting that the spin degeneracy is lifted through the Zeeman effect and the spin-up state (taking the fact that the $g$-factor is negative into account) moves up with increasing $B$. Here it is also clearly seen that a new peak is split out on the positive $V_{\mathrm{ds}}$ side and shifts to higher $V_{\mathrm{ds}}$ with increasing $B$. This split peak is associated with the transport through the Zeeman split spin-down state in the
QD. On the contrary, at $V_{\mathrm{bg}}=1.62 \mathrm{~V}$ (Fig. 3c), the QD is occupied by an odd number of electrons in the $\mathrm{CB}$ region and the pair of the inner $\mathrm{d} I_{\mathrm{ds}} / \mathrm{d} V_{\mathrm{ds}}$ peaks resemble the transport through the last occupied spin-up orbital level. When the magnetic field is applied, the two inner peaks move apart with increasing $B$. In this case, the two peaks do not exhibit splitting, confirming the half filling of an orbital state with a spin degeneracy of 2 . By fitting the peak shift in the low field region to the spin-1/2 Zeeman energy term $E_{Z}= \pm \frac{1}{2}\left|g^{*}\right| \mu_{\mathrm{B}} B$, where $\mu_{\mathrm{B}}$ is the Bohr magneton and $g^{*}$ is the effective $g$-factor, we can extract the $g$-factors associated with the two neighboring quantum levels as $\left|g_{\mathrm{A}}^{*}\right|=18$ and $\left|g_{\mathrm{B}}^{*}\right|=42$.

We can also determine the electron $g$-factors for different quantum levels from the magnetospectroscopy of the ground states of the quantum dot. ${ }^{8,33}$ Here we use the lock-in technique with a small excitation voltage of $5 \mu \mathrm{V}$ to measure the differential conductance $\mathrm{d} I_{\mathrm{ds}} / \mathrm{d} V_{\mathrm{ds}}$ as a function of $V_{\mathrm{bg}}$ and $B$ in a more positive gate voltage region containing three consecutive pairs of small and large $\mathrm{CB}$ diamonds or, equivalently, involving three neighboring quantum levels. Fig. 4 shows the results of the measurements where the evolutions of six consecutive Coulomb peaks with increasing $B$ are presented. As the field is increased, the conductance peaks move up or down, depending on the spin of the last occupied electron on the dot. Since the total ground-state spin state alternates between a singlet $S=0$ and a doublet $S=1 / 2$ in the zero and low magnetic field region, the peak spacing between a pair of a spin-up and a spin-down last occupied electron, after being converted from gate voltage to energy variation via the gate arm factor, reflects the Zeeman energy difference $\Delta E_{\mathrm{Z}}$. By fitting the measured data in the low field region to the Zeeman energy difference $\Delta E_{\mathrm{Z}}=\left|g^{*}\right| \mu_{\mathrm{B}} B$, we can obtain the effective $g$-factors for the three involved quantum levels. The results are $\left|g_{1}^{*}\right|=24,\left|g_{2}^{*}\right|=30$ and $\left|g_{3}^{*}\right|=25$. It is worth noting that in the large $B$ region shown in Fig. 4, the movements of the conductance peaks deviate from the linear magnetic field dependence. This is due to an increased influence of the magnetic field on the orbital states and the level repulsion in the

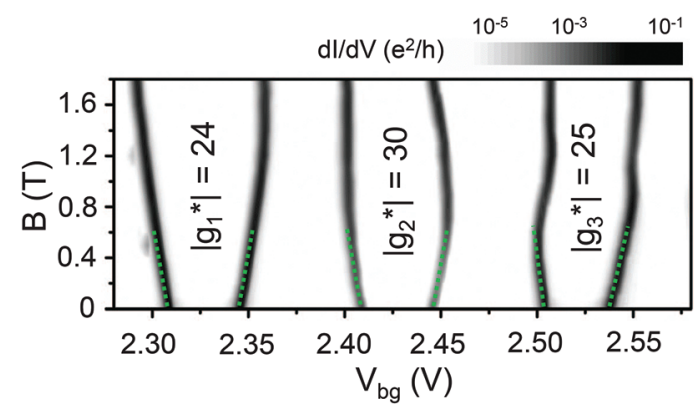

Fig. 4 Gray scale plot of magnetic field evolution of the differential conductance $\mathrm{d} l_{\mathrm{ds}} / \mathrm{d} V_{\mathrm{ds}}$ measured in the linear response regime (i.e., at bias voltage $V_{\mathrm{ds}}=0 \mathrm{~V}$ ) for the same quantum dot device as in Fig. 3. The $g$ factors extracted from the measurements for quantum levels are indicated in the figure. The green dashed lines denote the data used to extract the $g$-factors. 
presence of spin-orbit interaction (see below) at the crossover in ground states from a singlet to a triplet. ${ }^{34,35}$

Together with the values extracted from the energy spectra shown in Fig. 3, the $g$-factors in our MBE-grown InSb NW QDs show energy level-dependent fluctuations, which is consistent with previous reports on QD devices made from MOVPE-grown InSb NWs ${ }^{8}$ and MBE-grown InAs NWs. ${ }^{36}$ It has been known that level-to-level fluctuations of the $g$-factor in a QD imply the presence of spin-orbit coupling in the QD. ${ }^{37-39}$ We quantify the spin-orbit coupling strength in our InSb NW QDs by investigating the evolution of the excited state spectroscopy with increasing magnet field. The spin-orbit coupling mixes the Zeeman-split spin-up and spin-down states of different orbitals at a point of degeneracy and gives rise to level anticrossing. ${ }^{18,40,41}$ Fig. 5a shows the stability diagram of an MBEgrown InSb NW QD device with $\sim 50 \mathrm{~nm}$ in the NW diameter and $\sim 220 \mathrm{~nm}$ in the contact spacing. The CB diamonds are regularly spaced and show clear even-odd electron-number dependent alternations in size. From the measurements, we extract for the QD device an average charging energy $E_{\mathrm{C}}=$ $4.5 \mathrm{meV}$ and a quantum level spacing $\Delta \varepsilon=1.6 \mathrm{meV}$. Fig. $5 \mathrm{~b}$ shows the differential conductance spectrum measured along cut $\mathrm{A}$ in the stability diagram shown in Fig. $5 \mathrm{a}$ as a function of the magnetic field. The spin states of the two involved quantum levels (one in the ground state and the other one in the first excited state) are labeled in Fig. 5b. When the mag-
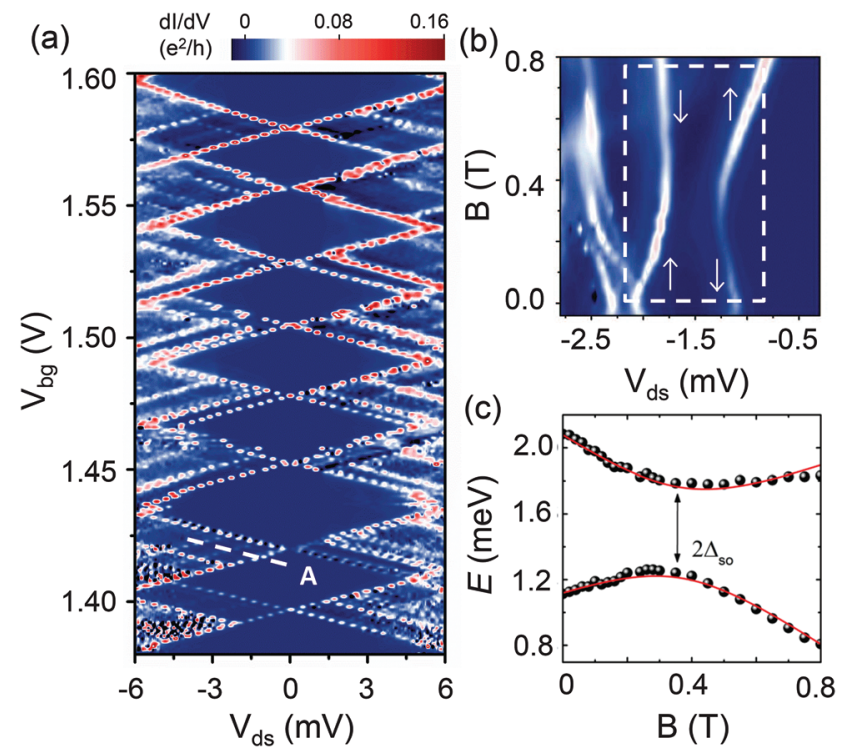

Fig. 5 Magnetic field evolutions of a ground state and a neighboring excited state of an InSb nanowire quantum dot device with a sourcedrain contact spacing of $\sim 220 \mathrm{~nm}$. (a) Charge stability diagram of the quantum dot at $B=0 \mathrm{~T}$. (b) Differential conductance measured along line cut $A$ in (a) as a function of $B$. (c) Converted energy positions of the differential conductance peaks in the region marked by the dashed rectangle in (b). The dots are the experimental energy positions of the peaks, the red lines are the results of a fit to a two-level perturbation model, and $\Delta_{\text {SO }}$ is the spin-orbit coupling strength assumed in the model, which causes the two quantum levels with different spins to undergo anti-crossing. netic field is increased, the spin-up and spin-down levels first move towards each other, then undergo an avoided crossing at $B \sim 0.4 \mathrm{~T}$, and finally move apart. It can be seen that the absolute values of the slopes corresponding the two levels are different, but remain almost unchanged after going through the avoided crossing region. The anti-crossing manifest an admixture of spin states near the degenerate point as a result of spin-orbit interaction. By fitting the energy positions of the two peaks to a simple two-level perturbation model, we can extract the spin-orbit coupling strength $\Delta_{\text {so }}$. The data points together with the best fit curves are displayed in Fig. 5c. Based on the fitting, we deduce a spin-orbit coupling strength $\Delta_{\text {So }} \approx$ $275 \mu \mathrm{eV}$ and a $g$-factor $\left|g^{*}\right|=27$ for the quantum level in the ground state and a $g$-factor $\left|g^{*}\right|=48$ for the quantum level in the excited state.

Finally, we would like to note that, considering the very high vacuum chamber and pure elemental sources used in the MBE growth, the coherent nature of electron transport through single InSb NW QDs demonstrated in the present work makes MBE-grown InSb nanowires attractive for further studies of many complex quantum transport phenomena. Especially, in the recent pursuit of Majorana bound states in semiconductor NWs, theoretical studies have proposed alternative interpretations for the experimental observations of zero-bias conductance peaks in superconductor-semiconductor NW hybrid devices and shown that a zero-bias conductance peak can occur in such hybrid devices with a moderate amount of disorder in the semiconductor NWs. ${ }^{42,43}$ In this regard, a clean and coherent semiconductor system, such as an MBE-grown InSb $\mathrm{NW}$, is desirable for the experimental detection of Majorana fermions in solid state. Furthermore, the realization of a fewelectron quantum system using such a clean and low-disorder InSb NW is important for exploring correlated electron phenomena at a low carrier density, such as the formation of a Wigner crystal at a one-dimensional quantum system, ${ }^{44-46}$ where the presence of only a small, finite number of electrons is crucial.

In conclusion, we have performed a detailed spectroscopic study of single QDs of different sizes made from MBE-grown InSb nanowires and have demonstrated coherent single-electron transport in the devices. The nanowires have a diameter of $50-80 \mathrm{~nm}$ and the QDs are defined in the nanowires between contacts by naturally formed Schottky barriers. Our results show that high-quality single QDs with a longitudinal size up to $700 \mathrm{~nm}$ can be realized using the MBE-grown InSb nanowires and the electron transport in the QDs can be controlled to occur in both the many-electron and the few-electron regime. The linear relationship between the gate capacitance and the source-drain electrode separation provides an experimental evidence for electron tunneling through quantum levels in single QDs. The measured $g$-factors are extracted to be in a range of 18-48 and show level-dependent fluctuations. A spin-orbit coupling strength of $\Delta_{\mathrm{SO}} \sim 275 \mu \mathrm{eV}$ is deduced by measuring the anticrossing of a ground state and a neighboring excited state. These results establish MBE-grown InSb NWs as a highly desired material platform for the further studies of strong-correlated many-body physics, including Majorana 
fermions in quantum nanostructures and for the development of high-performance quantum information devices.

\section{Conflict of Interest}

The authors declare no competing financial interests.

\section{Acknowledgements}

This work was supported by the National Basic Research Program of the Ministry of Science and Technology of China (Grants No. 2012CB932703 and No. 2012CB932700), the National Natural Science Foundation of China (Grants No. 11374019, No. 91221202, No. 91421303 and No. 61321001). N.K. thanks the Ph.D. Program Foundation of the Ministry of Education of China for financial support (Grant No. 20120001120126). H.Q.X acknowledges also the financial support from the Swedish Research Council (VR).

\section{References}

1 T. Jespersen, M. Aagesen, C. Sørensen, P. Lindelof and J. Nygård, Phys. Rev. B: Condens. Matter, 2006, 74, 233304.

2 A. V. Kretinin, H. Shtrikman, D. Goldhaber-Gordon, M. Hanl, A. Weichselbaum, J. von Delft, T. Costi and D. Mahalu, Phys. Rev. B: Condens. Matter, 2011, 84, 245316.

3 K. Hamaya, M. Kitabatake, K. Shibata, M. Jung, M. Kawamura, K. Hirakawa, T. Machida, T. Taniyama, S. Ishida and Y. Arakawa, Appl. Phys. Lett., 2007, 91, 232105.

4 A. Eichler, R. Deblock, M. Weiss, C. Karrasch, V. Meden, C. Schönenberger and H. Bouchiat, Phys. Rev. B: Condens. Matter, 2009, 79, 161407.

5 S. J. Chorley, M. R. Galpin, F. W. Jayatilaka, C. G. Smith, D. E. Logan and M. R. Buitelaar, Phys. Rev. Lett., 2012, 109, 156804.

6 S. Nadj-Perge, S. M. Frolov, E. P. A. M. Bakkers and L. P. Kouwenhoven, Nature, 2010, 468, 1084-1087.

7 Y. Hu, F. Kuemmeth, C. M. Lieber and C. M. Marcus, Nat. Nanotechnol., 2012, 7, 47-50.

8 H. A. Nilsson, P. Caroff, C. Thelander, M. Larsson, J. B. Wagner, L.-E. Wernersson, L. Samuelson and H. Q. Xu, Nano Lett., 2009, 9, 3151-3156.

9 H. A. Nilsson, O. Karlström, M. Larsson, P. Caroff, L. Pedersen, L. Samuelsom, A. Wacker, L.-E. Wernersson and H. Q. Xu, Phys. Rev. Lett., 2010, 104, 186804.

10 H. A. Nilsson, M. T. Deng, P. Caroff, C. Thelander, L. Samuelson, L.-E. Wernersson and H. Q. Xu, IEEE J. Sel. Top. Quantum Electron., 2011, 17, 907-914.

11 H. A. Nilsson, P. Samuelsson, P. Caroff and H. Q. Xu, Nano Lett., 2012, 12, 228-233.

12 S. Nadj-Perge, V. S. Pribiag, J. W. G. van den Berg, K. Zuo, S. R. Plissard, E. P. A. M. Bakkers, S. M. Frolov and L. P. Kouwenhoven, Phys. Rev. Lett., 2012, 108, 166801.
13 H. Yao, H. Y. Günel, C. Blömers, K. Weis, J. Chi, J. G. Lu, J. Liu, D. Grützmacher and T. Schäpers, Appl. Phys. Lett., 2012, 101, 082103.

14 I. van Weperen, S. R. Plissard, E. P. A. M. Bakkers, S. M. Frolov and L. P. Kouwenhoven, Nano Lett., 2013, 13, 387-391.

15 V. S. Pribiag, S. Nadj-Perge, S. M. Frolov, J. W. G. van den Berg, I. van Weperen, S. R. Plissard, E. P. A. M. Bakkers and L. P. Kouwenhoven, Nat. Nanotechnol., 2013, 8, 170-174.

16 S. R. Plissard, I. van Weperen, D. Car, M. A. Verheijen, G. W. G. Immink, J. Kammhuber, L. J. Cornelissen, D. B. Szombati, A. Geresdi, S. M. Frolov, L. P. Kouwenhoven and E. P. A. M. Bakkers, Nat. Nanotechnol., 2013, 8, 859-864.

17 D. Car, J. Wang, M. A. Verheijen, E. P. A. M. Bakkers and S. R. Plissard, Adv. Mater., 2014, 26, 4875-4879.

18 Ö. Gül, D. J. van Woerkom, I. van Weperen, D. Car, S. R. Plissard, E. P. A. M. Bakkers and L. P. Kouwenhoven, Nanotechnology, 2015, 26, 215202.

19 I. Vurgaftman, J. R. Meyer and L. R. Ram-Mohan, J. Appl. Phys., 2001, 89, 5815.

20 R. Isaacson, Phys. Rev., 1968, 169, 312-314.

21 V. Mourik, K. Zuo, S. M. Frolov, S. R. Plissard, E. P. A. M. Bakkers and L. P. Kouwenhoven, Science, 2012, 336, 1003-1007.

22 M. T. Deng, C. L. Yu, G. Y. Huang, M. Larsson, P. Caroff and H. Q. Xu, Nano Lett., 2012, 12, 6414-6419.

23 H. O. H. Churchill, V. Fatemi, K. Grove-Rasmussen, M. T. Deng, P. Caroff, H. Q. Xu and C. M. Marcus, Phys. Rev. B: Condens. Matter, 2013, 87, 241401.

24 M. T. Deng, C. L. Yu, G. Y. Huang, M. Larsson, P. Caroff and H. Q. Xu, Sci. Rep., 2014, 4, 7261.

25 C. Thelander, P. Caroff, S. R. Plissard and K. A. Dick, Appl. Phys. Lett., 2012, 100, 232105.

26 B. M. Borg and L.-E. Wernersson, Nanotechnology, 2013, 24, 202001.

27 T. Xu, K. A. Dick, S. Plissard, T. H. Nguyen, Y. Makoudi, M. Berthe, J.-P. Nys, X. Wallart, B. Grandidier and P. Caroff, Nanotechnology, 2012, 23, 095702.

28 D. B. Suyatin, C. Thelander, M. T. Björk, I. Maximov and L. Samuelson, Nanotechnology, 2007, 18, 105307.

29 Z. Zhong, Y. Fang, W. Lu and C. M. Lieber, Nano Lett., 2005, 5, 1143-1146.

30 W. Lu, J. Xiang, B. P. Timko, Y. Wu and C. M. Lieber, Proc. Natl. Acad. Sci. U. S. A., 2005, 102, 10046-10051.

31 L. L. Sohn, L. P. Kouwenhoven and G. Schön, Mesoscopic Electron Transport, Kluwer Dordecht, 1997.

32 O. Wunnicke, Appl. Phys. Lett., 2006, 89, 083102.

33 M. Björk, A. Fuhrer, A. Hansen, M. Larsson, L. Fröberg and L. Samuelson, Phys. Rev. B: Condens. Matter, 2005, 72, 201307.

34 D. Zumbühl, C. Marcus, M. Hanson and A. Gossard, Phys. Rev. Lett., 2004, 93, 256801.

35 R. Hanson, L. P. Kouwenhoven, J. R. Petta, S. Tarucha and L. M. K. Vandersypen, Rev. Mod. Phys., 2007, 79, 1217-1265.

36 S. Csonka, L. Hofstetter, F. Freitag, S. Oberholzer, C. Schönenberger, T. S. Jespersen, M. Aagesen and J. Nygard, Nano Lett., 2008, 8, 3932-3935. 
37 P. W. Brouwer, X. Waintal and B. I. Halperin, Phys. Rev. Lett., 2000, 85, 369-372.

38 K. A. Matveev, L. I. Glazman and A. I. Larkin, Phys. Rev. Lett., 2000, 85, 2789-2792.

39 D. A. Gorokhov and P. W. Brouwer, Phys. Rev. Lett., 2003, 91, 186602.

40 C. Fasth, A. Fuhrer, L. Samuelson, V. N. Golovach and D. Loss, Phys. Rev. Lett., 2007, 98, 266801.

41 D. Bulaev and D. Loss, Phys. Rev. B: Condens. Matter, 2005, 71, 205324.
42 J. Liu, A. C. Potter, K. T. Law and P. A. Lee, Phys. Rev. Lett., 2012, 109, 267002.

43 F. Pientka, G. Kells, A. Romito, P. W. Brouwer and F. von Oppen, Phys. Rev. Lett., 2012, 109, 227006.

44 L. H. Kristinsdóttir, J. C. Cremon, H. A. Nilsson, H. Q. Xu, L. Samuelson, H. Linke, A. Wacker and S. M. Reimann, Phys. Rev. B: Condens. Matter, 2011, 83, 041101.

45 V. V. Deshpande and M. Bockrath, Nat. Phys., 2008, 4, 314318.

46 K. A. Matveev, Phys. Rev. Lett., 2004, 92, 106801. 